\section{Experiencia terapéutica en un centro para la enfermedad de Chagas}

\author{
RAÚL ABRAMSON ${ }^{1}$, PATRICIA FIGUEROA ${ }^{2, a}$ \\ Therapeutic experience in a \\ Chagas disease center
}

\begin{abstract}
American Trypanosomiasis or Chagas Disease is a major public health problem, endemic in the American continent since prehistoric times. Its natural course is towards chronicity in the immunocompetent host, often leading to severe cardiopathy or bowel involvement. Pharmacologic therapy is restricted to two drugs and only one of them is currently available in Chile. Both have poor effectiveness in the chronic stages of the disease and cause frequent adverse reactions. Many physicians avoid their use, despite published evidences about the usefulness. We herein report the experience of our Center in the treatment of Chronic Chagas Disease in adults using the drug nifurtimox, emphasizing its degree of acceptability and its secondary effects.
\end{abstract}

(Rev Med Chile 2013; 141: 1427-1433)

Key words: Chagas disease; Chile; Drug toxicity; Nifurtimox.

'Departamento de Cardio-
logía y Unidad de Emer-
gencia Adultos, Hospital
Dr. Sótero del Río. Servicio
de Salud Metropolitano Sur
Oriente, Chile.
2a Enfermera Universitaria,
CDT Complejo Asistencial
Dr. Sótero del Río.
Declaración de conflictos
de Intereses: Los autores
declaran no tener ningún
tipo de relación contractual
ni de otro tipo con la
Industria Farmacéutica que
produce este fármaco o
cualquier otro.
Recibido el 3 de abril de
2013, aceptado el 22 de
octubre de 2013.
Correspondencia a:
Dr. Raúl Abramson
Departamento de
Cardiología.
Complejo Asistencial
Dr. Sótero del Río, Concha
y Toro 3459, Puente Alto,
Chile.
E-mail: rabr514@gmail.
com

L a Tripanosomiasis Americana, enfermedad descrita en 1909 por el médico brasileño Carlos Chagas y que lleva su nombre, es una zoonosis parasitaria vectorial causada por el protozoo flagelado Trypanosoma cruzi. Según evidencias arqueológicas, la enfermedad de Chagas (ECh) existe en las Américas desde hace más de 9.000 años y es endémica desde el sur de los Estados Unidos hasta la zona centro-sur de Chile y Argentina, con diferencias regionales. Su transmisión clásica es principalmente vectorial por insectos triatominos hematófagos del género Mepraia (Triatoma infestans y otras dos especies, nombre común: vinchuca) pero hoy predominan en nuestro país otras formas de transmisión: la transplacentaria ${ }^{1}$; a través de sangre contaminada; por trasplante de órganos infectados; por ingestión oral ${ }^{2}$ (no reportado en Chile) y otros mecanismos de importancia marginal. Al no existir una vacuna eficaz contra la enfermedad, las medidas de salud pública más importantes apuntan a contener los modos de transmisión. En Chile, desde 1999 está virtualmente interrumpida la transmisión por la vinchuca ${ }^{3}$ y el tamizaje a la sangre de dadores en los Bancos de Sangre del país mediante ELISA IgG es universal desde 2008. Sin embargo, la transmisión vertical sigue causando en Chile entre 500 y 1.000 recién nacidos infectados por T. cruzi al año, siendo hoy el principal mecanismo de transmisión.

En su evolución natural, en inmunocompetentes, la ECh cursa hacia la cronicidad. Pasa por 3 etapas: una breve etapa aguda, una etapa crónica indeterminada, latente o subclínica que suele durar décadas, $y$ finalmente puede haber una etapa crónica determinada, con manifestaciones clínicas características de la enfermedad. Estas afectan principalmente al corazón y al tubo digestivo, especialmente esófago y colon. En cualquier momento dado, aproximadamente $70 \%$ de los portadores del parásito están en la etapa crónica indeterminada o subclínica (en la que un tercio de ellos puede mantenerse toda la vida) y $30 \%$ tiene manifestaciones clínicas de daño. Esta cifra puede ser mayor, con métodos diagnósticos más sensibles. Se estima que en Chile anualmente $2 \%$ de los pacientes en etapa crónica indeterminada puede evolucionar a la etapa clínica, principalmen- 
te cardiopatía chagásica ${ }^{4}$. La inmunodepresión, de cualquier origen, puede causar importantes reactivaciones de la enfermedad.

Ninguna de las alternativas de tratamiento farmacológico disponibles se ha demostrado completamente eficaz en todas las formas de la enfermedad ${ }^{5}$. Los criterios de curación son controvertidos $^{6}$ y algunos expertos afirman que la ECh crónica hoy no puede ser curada ${ }^{7}$. Los estudios publicados son contradictorios y faltan estudios bien diseñados y de largo plazo. A pesar de ello existen muchas indicaciones que la terapia antiparasitaria es útil, con tasas variables de curación. La curación parasitológica y serológica en niños menores de dos años es prácticamente de $100 \%$. Pero, con los mismos criterios, la curación en adultos se estima en poco más de $30 \%$, ya que la serología convencional puede permanecer positiva por décadas aún en ausencia del parásito ${ }^{8}$. Sin embargo, hay consenso actual que la mayoría de los portadores del parásito se benefician si son tratados farmacológicamente, incluyendo pacientes ya dañados y que siguen albergando el parásito. Esto, porque hay evidencias que la disminución de la carga parasitaria influye favorablemente en la evolución de la enfermedad ${ }^{9-12}$. Por ello, los expertos concuerdan hoy en que toda persona con ECh debe ser tratada, en cualquiera de sus etapas, a excepción de los enfermos crónicos terminales ${ }^{8,11}$.

A pesar de estas recomendaciones, muchos pacientes no reciben el tratamiento farmacológico disponible para la ECh.

El propósito de este reporte es dar cuenta de la experiencia de nuestro centro acerca de la terapia de la ECh en adultos en etapa crónica con especial énfasis en su tolerancia y adhesividad al tratamiento con el fármaco disponible en Chile.

\section{Material y Método}

Este trabajo fue aprobado por el Comité de Ética del Servicio de Salud Metropolitano Sur Oriente.

El Centro de Chagas depende del Departamento de Cardiología y funciona en el Centro Diagnóstico Terapéutico (CDT) del Complejo Asistencial Dr. Sótero del Río del Servicio de Salud Metropolitano Sur Oriente. Comenzó sus funciones en 2009 y está a cargo de un Médico Internista y una Enfermera Universitaria. Es centro de derivación y cumple tareas clínicas y de consultoría, coordinándose con Epidemiología, Banco de Sangre y Laboratorio. Los pacientes son atendidos por el equipo médico-enfermera y luego de verificar su serología positiva para ECh el paciente es evaluado mediante clínica, laboratorio, radiografía de tórax y ECG, previo a ofrecerle el tratamiento etiológico correspondiente. La Reacción en Cadena de la Polimerasa (RCP) Trypanosoma cruzi cualitativa se efectúa en el Laboratorio de Biología Molecular, Sección Parasitología del Instituto de Salud Pública de Chile, con Técnica RCP convencional internacionalmente aceptada ${ }^{13}$, utilizando los partidores 121-122 que tienen como secuencia blanco el kinetoplasto del Trypanosoma cruzi. La serología convencional (ELISA IgG e Inmunofluorescencia indirecta IFI) se hace en el Laboratorio de Parasitología Clínica, Sección Parasitología del mismo Instituto usando antígenos solubles para ELISA y figurado para IFI, obtenido de cultivo in vitro de la cepa Tulahuén, origen chileno. Hacemos una encuesta epidemiológica en todos los pacientes, con especial atención a la mujer chagásica. El tratamiento es detalladamente explicado, incluyendo eventuales efectos secundarios. Sólo se excluye del tratamiento farmacológico a pacientes con contraindicación formal: alcoholismo, cardiopatía terminal, pacientes muy añosos con daños avanzados, portadores de megaesófago con dificultad de deglución. Tampoco se trata a embarazadas y pacientes con falla renal o hepática avanzada. Los pacientes que completan su tratamiento son citados un año después de éste y luego anualmente, para su evaluación.

La Tabla 1 resume las actividades del centro en el período analizado.

La Tabla 2 describe las características y origen de la población atendida.

La terapia de la ECh se basa en fármacos tripanomicidas. Los fármacos clásicos, únicos aprobados hasta ahora para el tratamiento de la enfermedad de Chagas, son un nitrofurano, Nifurtimox (NFX) y un nitroimidazol, Benznidazol (BNZ). Se han incorporado alternativas de efectividad discutida y en estudio,como itraconazol, alopurinol y otras ${ }^{8,14-16}$, en especial en adultos en fase crónica y en casos especiales, como trasplante de órganos. En el país no se encuentra habitualmente disponible BNZ. Está actualmente en desarrollo un importante estudio multicéntrico sobre la eficacia de BNZ en cardiopatía chagásica ${ }^{17}$. El nifurtimox 
Experiencia terapéutica en un centro de Chagas - R. Abramson et al

(Lampit ${ }^{\circledR}$ Bayer), único fármaco generalmente usado en Chile y con el cual hemos hecho nuestra experiencia, fue desarrollado en 1960. El BNZ (Rochagan ${ }^{\circledR}$ o Radanil ${ }^{\circledR}$ Roche) apareció en 1974. Hasta la fecha no se han desarrollado nuevos fármacos específicos, lo que se ha atribuido a desinterés de la industria farmacéutica ("enfermedades desatendidas") ${ }^{18,19}$.

Así como en Chile no se dispone de BNZ, en diversos países no se usa NFX. Esto obedece a los efectos secundarios que ambos fármacos presen-

Tabla 1. Actividades del Centro de Chagas a agosto 2012

\begin{tabular}{|lr|}
\hline Total de pacientes & $\mathbf{2 0 2}$ \\
\hline Pacientes tratados & 142 \\
\hline Pacientes tratados con control a un año & 91 \\
\hline Pacientes tratados con control a dos años & 12 \\
\hline Pendiente control del primer año & 39 \\
\hline En tratamiento & 6 \\
\hline Tratamiento pendiente & 8 \\
\hline Tratamientos suspendidos* & 2 \\
\hline No ingresados a tratamiento** & 30 \\
\hline
\end{tabular}

*Por efectos adversos. **Contraindicaciones, abandonos, inasistentes, rechazo a ser tratado, traslados, inubicables, fallecidos.

Tabla 2. Características de los pacientes

\begin{tabular}{|lr|}
\hline Edad años (promedio y rango) & $48,9(17-82)$ \\
Sexo masculino & $99(49 \%)$ \\
Sexo femenino & $103 \quad(51 \%)$ \\
Antecedentes epidemiológicos & $60(29,7 \%)$ \\
identificados & \\
Etapa ECh al momento del tratamiento \\
Etapa aguda & 0 \\
Etapa crónica indeterminada & $160(79,2 \%)$ \\
Etapa crónica determinada & $24(11,9 \%)$ \\
En estudio & $18(8,9 \%)$ \\
Origen de los pacientes & \multicolumn{2}{c}{} \\
Bancos de sangre & $118(58,4 \%)$ \\
Traslado de otros centros & $38(18,8 \%)$ \\
Especialidades & $33(16,3 \%)$ \\
Atención primaria & $6(2,9 \%)$ \\
Otros & $7(3,5 \%)$ \\
\hline
\end{tabular}

ECh: Enfermedad de Chagas. $\tan$ y a diferencias de eficacia observadas, que probablemente están en relación a distintas cepas de Trypanosoma cruzi $^{20}$. El NFX se ha demostrado más eficaz en Chile que en otros países de la región. Está disponible en el sistema público de salud y se presenta en frascos de 100 comprimidos de 120 $\mathrm{mg}$. Aunque el fármaco es antiguo el fabricante ha hecho recientes mejoras farmacotécnicas que han disminuido los efectos secundarios ${ }^{8}$. Se administra en dosis de $6-8 \mathrm{mg} / \mathrm{kg}$ en adultos y $10 \mathrm{mg} / \mathrm{kg}$ en niños, dividido en 3 dosis diarias después de alimentos, durante 60 días. Iniciamos el tratamiento a $1 / 4$ ó $1 / 3$ de la dosis calculada y lo aumentamos gradualmente en 4 a 7 días. Controlamos los pacientes con evaluación clínica y exámenes de laboratorio en promedio 3 veces (durante el tratamiento y al completar éste) con especial atención a los posibles efectos secundarios. Los análisis incluyen hemograma, VHS, perfil hepático, perfil bioquímico, proteína $\mathrm{C}$ reactiva y creatinakinasa.

La tolerancia suele ser buena en niños ${ }^{21}$. En adultos es esperable la aparición de reacciones adversas en aproximadamente $30 \%$, relacionadas con su mecanismo de acción ${ }^{5,8,22}$.

\section{Resultados}

La Tabla 3 muestra nuestros resultados del tratamiento, medido con RCP Trypanosoma cruzi y serología convencional en los pacientes controlados después de un año de terminado su tratamiento farmacológico.

La Tabla 4 describe las reacciones reportadas en la literatura para todos los grupos de edad en series de casos y ensayos clínicos, usando dosis estándar ${ }^{7}$.

En la Tabla 5 se muestra nuestros pacientes tratados y la aparición de efectos secundarios en

\section{Tabla 3. Control de RCP y Serología en 88 pacientes un año post-tratamiento*}

\begin{tabular}{|lcc|}
\hline Prueba & $\begin{array}{c}\text { Positivo } \\
\text { n (\%) }\end{array}$ & $\begin{array}{c}\text { No detectable } \\
\text { n (\%) }\end{array}$ \\
\hline RCP Trypanosoma cruzi & $12(13,6)$ & $76(86,4)$ \\
\hline Serología & Positiva & Negativa \\
\hline ELISA IgG & $87(98,9)$ & $1(1,1)$ \\
IFI & $87(98,9)$ & $1(1,1)$ \\
\hline
\end{tabular}

*3 resultados pendientes. $\mathrm{RCP}$ : Reacción en Cadena de la Polimerasa. IFI: Inmunofluorescencia Indirecta. 
ese grupo.

En la Tabla 6 los efectos secundarios se agrupan según su intensidad o gravedad. Consideramos tolerancia "muy buena" la ausencia de molestias reportadas, "buena" efectos menores que no in-

Tabla 4. Efectos adversos de NFX descritos en la literatura

\begin{tabular}{|c|c|}
\hline Efectos adversos & $\begin{array}{c}\% \\
\text { aproximado } \\
\text { (rango) }\end{array}$ \\
\hline Anorexia y baja de peso & $50(17-70)$ \\
\hline Polineuropatía (dosis dependiente) & $25(15-36)$ \\
\hline Náuseas, vómitos & $15(5-33)$ \\
\hline Dolor abdominal & $15(9-25)$ \\
\hline Cefalea & $15(13-19)$ \\
\hline Mareos, vértigo & $15(9-25)$ \\
\hline Insomnio & $12(10-19)$ \\
\hline Irritabilidad, cambios de carácter & $15(9-27)$ \\
\hline Mialgias & $12(11-14)$ \\
\hline Desorientación, alteraciones de memoria & $7(6-10)$ \\
\hline Parestesias & $3(2-3)$ \\
\hline Alteraciones cutáneas & $(1-4)$ \\
\hline Leucopenia & $<3$ \\
\hline
\end{tabular}

NFX: Nifurtimox.

Tabla 5. Efectos adversos observados en 142 pacientes tratados con nifurtimox

\begin{tabular}{|lrr|}
\hline Efectos adversos observados & n & (\%) \\
\hline Baja de peso & 112 & $(78,9)$ \\
\hline Anorexia & 65 & $(45,7)$ \\
Náuseas, vómitos, dolor abdominal & 49 & $(34,5)$ \\
Mialgias, lumbalgia, astenia & 38 & $(26,7)$ \\
Cefalea & 19 & $(13,3)$ \\
Insomnio & 17 & $(11,9)$ \\
Irritabilidad, cambios de carácter & 3 & $(2,1)$ \\
Alteraciones cutáneas (rash severo en & 3 & $(2,1)$ \\
dos casos) & 2 & $(1,4)$ \\
Mareos, vértigo & 2 & $(1,4)$ \\
Desorientación, alteraciones de memoria & 0 & \\
Parestesias & 0 & \\
Leucopenia & 0 & \\
\hline Polineuropatía & & \\
\hline
\end{tabular}

terfieren con el cumplimiento, "regular" efectos secundarios que motivaron quejas significativas o que obligaron a hacer una pausa en el tratamiento (reanudándolo y terminándolo después) y "mala" efectos secundarios que obligaron a la interrupción definitiva del tratamiento. Los resultados de los exámenes de laboratorio no influyeron en esta clasificación dado que en $92,6 \%$ de los pacientes se mantuvieron en límites normales durante toda la evaluación y ninguno de ellos presentó alteraciones mayores, como se explica más abajo.

No hay acuerdo sobre los criterios de curación de la enfermedad. Si bien en el período agudo se puede obtener curación clínica, serológica y parasitológica, en el período crónico esto no ocurre. La serología convencional puede permanecer positiva por décadas, en ausencia del parásito, lo que se ha atribuido a memoria inmunológica. Sin embargo un método directo, como es la RCP Trypanosoma cruzi, se considera un indicador mucho más preciso de la presencia del parásito. Los niveles de sensibilidad pueden ser variables pero su especificidad es cercana a $100 \% 0^{1,23}$ y nuevas técnicas tienen sensibilidad y especificidad casi $100 \%{ }^{24}$. Por consenso, los criterios de curación más aceptados son la negativización de los exámenes parasitológicos directos, la RCP Trypanosoma cruzi no detectable (que también es un método directo), la disminución progresiva de los títulos serológicos y la remisión de las alteraciones electrocardiográficas, todo lo que debe mantenerse en el tiempo. Se recomienda evaluar al paciente un año después de su tratamiento y en años consecutivos. En ese momento estudiamos su estado clínico, ECG, serología convencional y RCP cualitativoTrypanosoma cruzi. En nuestro centro no utilizamos el xenodiagnóstico porque en pacientes crónicos los falsos negativos alcanzan entre $40-80 \%{ }^{4,25}$.

Si bien la serología convencional, medida a un

Tabla 6. Grado de tolerancia al tratamiento observada en 142 pacientes tratados con nifurtimox

\begin{tabular}{|lrr|}
\hline Tolerancia observada & n & (\%) \\
\hline Muy buena & 11 & $(7,7)$ \\
\hline Buena & 73 & $(51,4)$ \\
\hline Regular & 49 & $(34,5)$ \\
\hline Mala & 3 & $(2,1)$ \\
\hline Sin información & 6 & $(4,2)$ \\
\hline
\end{tabular}


año del tratamiento, continuó positiva en $98,9 \%$ de nuestros pacientes (aunque en general a títulos menores, como ha sido ampliamente descrito en la literatura) $86,4 \%$ de éstos a un año del tratamiento mostraron RCP específico para T. cruzi no detectable. En esta cohorte no dispusimos de RCP previo a la farmacoterapia por lo que no podemos sacar conclusiones de este dato, ya que ignoramos cuántos eran no detectables previamente.

$\mathrm{La}$ adhesividad al tratamiento fue muy alta, completándose los dos meses en 97,9\%.En cuanto al aspecto más discutido del tratamiento, la tolerancia observada al NFX fue "muy buena" o "buena" en 59,1\% de los pacientes. Aproximadamente $37 \%$ de los pacientes reportó algún efecto secundario significativo que en su mayoría fueron de carácter moderado y sólo $2,1 \%$ no pudo completarlo por efectos adversos inaceptables. Frecuentemente los efectos secundarios fueron más notorios al inicio del tratamiento y se atenuaron después. El efecto observado con mayor frecuencia fue la baja de peso (hasta $5 \mathrm{~kg}$ ), sólo en parte relacionada con anorexia. Sigue en frecuencia las molestias digestivas como dispepsia, náuseas o vómitos y luego molestias corporales inespecíficas. Catorce por ciento de los pacientes relató cefalea de intensidad variable y un porcentaje similar reportó insomnio, irritabilidad o ansiedad, lo que puede o no estar relacionado con el fármaco. Los 2 pacientes en que debimos interrumpir definitivamente el tratamiento presentaron un rash cutáneo importante de tipo medicamentoso. En ambos casos el rash cedió en pocos días al dejar el fármaco. Por otra parte una paciente acusó pérdida de memoria, por lo cual dejó el tratamiento una semana antes de terminarlo. Otra paciente joven, asintomática previamente, presentó convulsiones 4 días antes de completar el tratamiento, el que había hecho sin molestias o incidentes hasta entonces. Los neurólogos opinaron que se trata de un cuadro comicial genuino, probablemente no relacionado al fármaco. Incidentalmente, estas dos últimas pacientes presentaron RCP T. cruzi no detectable, al ser controladas un año después. En la totalidad de los casos observados las molestias secundarias, de cualquier grado, desaparecieron en alrededor de una semana al terminar el tratamiento. En los pacientes controlados un año después se observó también recuperación del peso corporal perdido en el tratamiento.Todos los pacientes que estaban en etapa crónica indeterminada al iniciar el tra- tamiento $(\mathrm{n}=160)$ continuaban en ese estado un año después.

En 92,6\% de los pacientes los exámenes de laboratorio se mantuvieron en límites normales durante toda la evaluación. Cuatro por ciento de los pacientes presentaron leucocitosis (inferior a $14.000 / \mathrm{mL}$ ) y ninguno presentó leucopenia. Otro $4 \%$ mostró proteína $\mathrm{C}$ reactiva discretamente aumentada, hasta 22. Una paciente tuvo alteración significativa de las pruebas hepáticas, coincidente con un cuadro biliar incidental.

A diferencia de lo reportado en la literatura (Tabla 3) en nuestro grupo no observamos ningún caso de polineuropatía. Probablemente esto se debe a que la reacción es dosis dependiente y nuestra dosis promedio fue 6 a $7 \mathrm{mg} / \mathrm{kg}$ de peso y a que es más común en tratamientos prolongados, mayores a 60 días. Por otra parte, tuvimos más casos de anorexia, baja de peso y molestias digestivas que las reportadas.

\section{Discusión}

A pesar de que existen en Latino América más de 15 millones de pacientes infectados de ECh (OMS, 2000 y 2006), que entre 80 y 100 millones de personas están en riesgo de contraerla y que esta enfermedad ocupa actualmente el cuarto lugar de importancia como carga de enfermedad medida en años de vida ajustados según discapacidad (AVAD) en América, después de las infecciones respiratorias, gastrointestinales y SIDA $^{4,11}$, sólo hay dos fármacos para tratarla, el más nuevo de los cuales ya tiene casi 40 años en el mercado. Ha habido evidente desinterés de la industria farmacéutica por esta "enfermedad desatendida"26,27. Ambos fármacos son de efectividad limitada en el paciente crónico y tienen notorios efectos secundarios en adultos. Pero, con las controversias que haya sobre las tasas de curación, la recomendación de tratar a estos pacientes está bien fundamentada y debemos usar lo que está disponible. Por ello, es importante tener datos objetivos sobre su tolerancia y aceptabilidad, en nuestro medio. Muchos portadores de ECh no reciben terapia farmacológica a pesar de las recomendaciones. Nuestra experiencia hasta ahora no avala esta actitud.

Es indudable que los efectos secundarios del NFX son frecuentes. Sin embargo, el uso clínico de medicamentos útiles pero con perfil potencial- 
mente desfavorable, ya sea por su margen terapéutico estrecho o por conocidos eventos negativos, es habitual en la práctica médica.

A pesar del perfil en general alentador observado en nuestros pacientes, sólo su seguimiento por muchos años puede demostrar una eventual "curación" de la enfermedad, al no aparecer las complicaciones cardíacas o digestivas. Esta cohorte debiera ser seguida anualmente con evaluación clínica, serología, RCP T. cruzi, ECG y ecocardiografía para determinar la real efectividad del fármaco. Nuestra nueva cohorte es estudiada con RCP $T$. cruzi pre y post tratamiento, para evaluar la tasa de negativización. De acuerdo al conocimiento actual y a la espera de nuevas evidencias o estrategias de tratamiento, creemos que la disminución de la carga parasitaria, sugerida por la RCP T. cruzi no detectable, puede representar lo más próximo a curación de la ECh crónica que hoy se puede alcanzar con la terapia disponible.

Agradecimientos: A Karina González Tello, Secretaria de Programas de Enfermedades Emergentes, Complejo Asistencial Dr. Sótero del Río, por su colaboración en la recolección de datos.

\section{Referencias}

1. García A, Bahamonde MI, Verdugo S, Correa J, Pastene C, Solari A et al. Infección transplacentaria por Trypanosoma cruzi: Situación en Chile. Rev Med Chile 2001; 129 (3): 330-2

2. Toso A, Vial F, Galanti N. Transmisión de la enfermedad de Chagas por vía oral. Rev Med Chile 2011; 139: 25866.

3. Lorca M, García A, Bahamonde MI, Fritz A, Tassara R. Certificación serológica de la interrupción de la transmisión vectorial de la enfermedad de Chagas en Chile. Rev Med Chile 2001; 129: 266-71.

4. Apt W, Heitmann I, Jercic MI, Jofré L, Muñoz P, Noemí I, et al. Guías de Diagnóstico, Tratamiento y Prevención de la Enfermedad de Chagas. Santiago, MINSAL 2010. Rev Chil Infectol 2008; 25; 189-93; 194-99; 285-8; 289-92; 378-83; 384-9.

5. Storino R, Gallerano R, Sosa R. Tratamiento Antiparasitario Específico, en: Enfermedad de Chagas, Storino R. Milei J; Doyma, Editor. 1993, Barcelona. p. 557-69.

6. Fuentes BR, Maturana AM, de la Cruz MR. Eficacia de nifurtimox para el tratamiento de pacientes con enfermedad de Chagas crónica. Rev Chil Infect 2012; 29 (1):
82-6.

7. UptoDate; Chagas Disease: Management of Acute Disease, Early Chronic Disease, and Disease in Inmunocompromised Hosts. [Accessed on June 04, 2013].

8. Apt W, Zulantay I. Estado actual en el tratamiento de la enfermedad de Chagas. Rev Med Chile 2011; 139: 247 57.

9. Bern C, Montgomery SP, Herwaldt BL, Rassi A Jr, MarínNeto JA, Dantas RO, et al. Evaluation and treatment of Chagas disease in the United States: a systematic review. JAMA 2007; 298 : 2171-81.

10. Rassi A Jr, Dias JC, Marín-Neto J, Rassi A. Challenges and opportunities for primary, secondary, and tertiary prevention of Chagas' disease. Heart 2009; 95: 524-34.

11. Control of Chagas disease. WHO Technical Report Series 2000; 905 Geneva.

12. Teixeira A, Nascimento R, Sturn N. Evolution and pathology in Chagas disease. A Review. Mem Inst Oswaldo Cruz 2006; 101: 463-91.

13. Schijman AG, Bisio M, Orellana L, Sued M, Duffy T, Mejia Jaramillo AM, et al. International study to evaluate PCR methods for detection of Trypanosoma cruzi DNA in blood samples from Chagas disease patients. PLoSNegl Trop Dis 2011; 5 (1): e931. doi: 10.1371/journal. pntd.0000931. [Accessed on June 20, 2013].

14. Apt W, Aguilera A, Arribada A, Perez C, Miranda C, Sanchez G, et al. Treatment of Chronic Chagas' Disease with Itraconazole and Allopurinol. Am J Trop Med Hyg 1998; 59 (1): 133-8.

15. Rassi A, Ostermayer A, Rassi Jr A, Garcia da Silva, et al. Specific Treatment ForTrypanosoma Cruzi: Lack Of Efficacy Of Allopurinol In The Human Chronic Phase Of Chagas Disease. Am J Trop Med Hyg 2007; 76 (1): 58-61.

16. Almeida DR, Carvalho AC, Branco JN, Pereira AP, Correa L, Vianna PV, et al. Chagas' disease reactivation after heart transplantation: efficacy of allopurinol treatment. J Heart Lung Transplant 1996; 15 (10): 988-92.

17. Marín-Neto JA, RassiJr A, Morillo CA, Avezum A, Connolly S, Sosa-Estani S, et al. Rationale and design of a randomized placebo-controlled trial assessing the effects of etiologic treatment in Chagas' cardiomyopathy: The BENznidazole Evaluation For Interrupting Trypanosomiasis (BENEFIT). Am Heart J 2008; 156: 37-43.

18. Drugs for Neglected Disease Initiative. Chagas disease: DNDi Strategy, 2010. http://www.treatchagas.org/ rd_dndi_strategy.aspx [Accessed on August 22, 2012].

19. Oprea L, Braunack-Mayer A, Gericke CA. Ethical issues in funding research and development of drugs for neglected tropical diseases. J Med Ethics 2009; 35: 310-4. [Downloaded from jme.bmj.comon August 22, 2012] 
Experiencia terapéutica en un centro de Chagas - R. Abramson et al

20. de Meis J, Morrot A, Farias-de-Oliveira DA, VillaVerde D M, Savino W. Differential regional immune response in Chagas disease. PLoSNeglTropDis 2009; 3: e417. Disponible enhttp://www.plosntds.org/article/ info:doi/10.1371/journal.pntd.0000417 [Accessed June 04, 2013].

21. Schenone H, Contreras M, Solari A, García A, Rojas A, Lorca M. Tratamiento con Nifurtimox de la infección chagásica crónica en niños. Rev Med Chile 2003; 131: 1089-90.

22. Castro JA, Díaz de Toranzo EG. Toxic effects of nifurtimox and benznidazole, two drugs used against American trypanosomiasis (Chagas Disease). Biomed Environ Sci 1988; 1: 19-33.

23. Russomando G, De Tommassone M, De Guillen I, Acosta N, Vera N, Almiron M, et al. Treatment of congenital Chagas disease diagnosed and followed up by the polymerase chain reaction. Am J Trop Med Hyg 1998; 59:
487-91.

24. Deborggraeve S, Coronado X, Solari A, Zulantay I, Apt W, Mertens P, et al. T. cruzi OligoC- TesT: A Simplified and Standardized Polymerase Chain Reaction Format for Diagnosis of Chagas Disease. PLoS Negl Trop Dis 2009; 3 (6): e450. Disponible en http://www.plosntds. org/article/info:doi/10.1371/journal.pntd.0000450 [Accessed on June 04, 2013].

25. Castro C, Macedo V, Prata A. The behavior of Trypanosoma cruzi parasitemia in chronic chagasics over 13 years. Rev Soc Bras Med Trop 1999; 32: 157 65.

26. Urbina JA. New advances in the management of a longneglected disease. Clin Infect Dis 2009; 49: 1685.

27. Mathers CD, Ezzati M, López AD. Measuring the burden of neglected tropical diseases: the global burden of disease framework. Disponible en PLoSNegl Trop Dis 2007; 1: e114 [Accessed on June 04, 2013]. 